\title{
Composición corporal y metabolismo energético en mujeres con exceso de peso
}

\author{
Body composition and energy metabolism in women \\ with excess weight
}

\author{
E. Lopes Rosado ${ }^{1}$, J. Bressan ${ }^{2}$, C.L. D’Andrea ${ }^{1}$, C.E. Vogel $^{1}$
}

\section{RESUMEN}

Fundamentos. El objetivo de presente trabajo es evaluar la composición corporal y el metabolismo energético en mujeres con exceso de peso corporal.

Material y métodos. Estudio transversal de intervención no randomizado con 40 mujeres [ 26 con exceso de peso (G1) y 14 eutróficas (G2)]. Fueron realizadas evaluaciones dietéticas, antropométricas y de la composición corporal (bioimpedancia eléctrica), actividad física (acelerómetro tridimensional) y metabolismo energético (calorimetría indirecta).

Resultados. No hubo diferencia en la ingesta energética y en la actividad física entre los grupos. Los parámetros de composición corporal fueron superiores en G1, excepto el agua corporal total. Hubo asociación entre el gasto energético y la composición corporal. La masa corporal magra fue el principal determinante del gasto energético. No hubo diferencia de los parámetros metabólicos entre los grupos, pero se sugiere menor velocidad de oxidación de los nutrientes y mayor eficiencia metabólica en G1.

Conclusiones. El exceso de peso corporal se asoció con cambios en la composición corporal y en el metabolismo energético que justifican la acumulación de grasa corporal.

Palabras clave. Obesidad. Composición corporal. Metabolismo energético. Dieta.

\begin{abstract}
Background. The objective of this paper is to evaluate body composition and energy expenditure in women with excess body weight.

Methods. There was a non-randomized, cross-sectional study with 40 women, [ 26 with excess weight (G1) and 14 eutrophic (G2)]. The following evaluations were made: dietetic, anthropometric and body composition (electrical bioimpedance), physical activity (threedimensional accelerometer) and energy expenditure, basal and resting (indirect calorimetry).
\end{abstract}

Results. The energy intake and physical activity did not differ between groups. The parameters of body composition were higher in G1, except total body water. There was a relationship between energy expenditure and body composition. The lean mass was the biggest determinant of energy expenditure. There was no difference in metabolic parameters between groups, but lower nutrient oxidation and increased metabolic efficiency in G1 was suggested.

Conclusion. Excess weight was associated with body composition and energy expenditure changes that justify the accumulation of body fat.

Key words. Obesity. Body composition. Energy metabolism. Diet.
1. Departamento de Nutrição e Dietética. Instituto de Nutrição Josué de Castro. Universidade Federal do Rio de Janeiro-RJ.

2. Departamento de Nutrição. Universidad Federal de Viçosa-MG.

Recepción el 10 de marzo de 2010

Aceptación provisional el 15 de abril de 2010

Aceptación definitiva el 7 de mayo de 2010

\section{Correspondencia}

Eliane Lopes Rosado

Departamento de Nutrição e Dietética

Instituto de Nutrição

Universidade Federal do Rio de Janeiro

Avda. Brigadeiro Trompowski, s/n. Edificio do

CCS, bloco J, $2^{\circ}$ andar

Cidade Universitaria. Ilha do Fundão CEP: 21941-590 Rio de Janeiro. Brasil

E-mail: elianerosado@nutricao.ufrj.br 


\section{INTRODUCCIÓN}

La obesidad es una enfermedad multifactorial que se caracteriza por el desequilibrio energético debido al exceso en la ingesta energética y/o reducción del gasto energético ${ }^{1}$.

El gasto de energía está relacionado con la oxidación del sustrato, que es determinada por la velocidad en que la adenosina trifosfato (ATP) es utilizada, lo que se relaciona con el tamaño corporal y la actividad física, siendo diferente en diferentes tejidos corporales ${ }^{2}$.

En este sentido, la composición corporal y la oxidación de los nutrientes, en particular de los lípidos, son importantes en el origen de la obesidad. La relación entre la masa grasa (MG) y la masa libre de grasa (MLG) es mayor en obesos, siendo la MG menos activa metabólicamente ${ }^{3}$ y la cantidad y la distribución de la misma influye en la oxidación de lípidos. Algunos estudios informan sobre la reducción de la oxidación de los lípidos ${ }^{3,4} \mathrm{y}$ de un aumento del almacenamiento de triglicéridos y ácidos grasos libres ${ }^{5}$ en sujetos obesos, en comparación con aquellos de peso normal.

Se sugiere que la MLG es la principal responsable del aumento de la oxidación de los lípidos ${ }^{6,7}$ ya que los procesos bioquí-

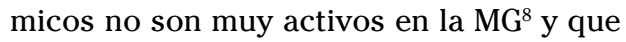
el tejido muscular tiende a mantener su metabolismo más activo, incluso durante el reposo ${ }^{9}$. Sin embargo, Astrup y col ${ }^{10}$ evaluaron a mujeres con sobrepeso/obesidad y eutróficas y observaron una correlación positiva entre la MG y la oxidación de los lípidos durante 24 horas.

La evaluación del gasto de energía, y en particular de los componentes relacionados con la oxidación de sustratos, es importante en los estudios relacionados con la obesidad ${ }^{11}$.

La tasa de oxidación de sustratos puede ser evaluada por el cociente respiratorio (CR), que depende de la mezcla de combustibles que se metaboliza, siendo igual a 1,0 para los hidratos de carbono ya que el número de moléculas de dióxido de carbono $\left(\mathrm{CO}_{2}\right)$ producido es igual al número de moléculas de oxígeno $\left(\mathrm{O}_{2}\right)$ consumido, y para los lípidos, las proteínas y la dieta mixta, los valores de CR son de 0,70, 0,82 y 0,85 , respectivamente ${ }^{12}$. Sin embargo, Westerterp-Plantenga y col $^{13}$ consideran que la composición de la dieta no influye en el gasto de energía.

Además el gasto energético en reposo (GER) en las personas obesas es más alto en comparación con las personas de peso normal; sin embargo, la tasa metabólica basal (TMB), expresada como el gasto de energía por kilogramo de peso corporal en un tiempo determinado, es inferior ${ }^{14}$. Para Censi y col $^{15}$, la tasa metabólica depende de la cantidad y de la actividad metabólica de la masa celular corporal, así como su relación con la MLG. Por lo tanto, las personas obesas requieren mayores cantidades de energía para mantener su peso corporal por presentar mayor masa corporal total. Sin embargo, tienen bajos requerimientos de energía, teniéndose en cuenta la TMB.

La TMB ( $\mathrm{kcal} / \mathrm{min} / \mathrm{kg}$ de peso corporal) y la tasa metabólica en reposo (TMR) se refieren al metabolismo del cuerpo durante el ayuno y en el período postabsortivo, respectivamente. La TMR incluye los gastos para la digestión, absorción y distribución corporal de los nutrientes ingeridos. En la mayoría de los adultos sedentarios la TMB constituye aproximadamente un $60 \%$ al $70 \%$ del gasto energético diario; sin embargo, puede variar con el tamaño y la composición corporal. La MLG, MG, edad y sexo son los principales determinantes de la TMB, representando un $80 \%$ de la variación de la misma ${ }^{16}$.

La tasa metabólica puede ser cuantificada mediante la medición del consumo de $\mathrm{O}_{2}$, producción de $\mathrm{CO}_{2}$ y la excreción urinaria de nitrógeno, con el individuo despierto o en reposo, en ambiente y con temperatura neutra, después del ayuno nocturno de 8 a 12 horas $^{16}$. El volumen de oxígeno consumido $\left(\mathrm{VO}_{2}\right)$ está directamente relacionado con el tamaño del cuerpo $^{17}$, mientras que el volumen de dióxido de carbono producido $\left(\mathrm{VCO}_{2}\right)$ está relacionado con el tipo de nutriente oxidado y la eficiencia metabólica ${ }^{13}$. 
Según Valtueña y $\operatorname{col}^{14}$, los factores involucrados en el aumento de peso corporal, identificados en los estudios longitudinales en poblaciones con tendencia a la obesidad, incluyen la baja tasa metabólica y el alto CR. El CR elevado muestra gran tendencia a ahorrar energía y, consecuentemente, mayor eficiencia metabólica ${ }^{3,18}$. Marra y coll $^{19}$ verificaron una mayor TMR, ajustada por la MLG, en las mujeres obesas en comparación con las eutróficas. Katzmarzyk y $\mathrm{col}^{20}$ no observaron relación entre la TMR y el CR con la masa corporal. Cabe señalar que los estudios que relacionan la tasa metabólica y la masa corporal no son concluyentes ${ }^{18}$.

El presente estudio tiene como objetivo evaluar la composición corporal y el metabolismo energético, así como la relación entre estas variables, en las mujeres con exceso de masa corporal y eutróficas, ya que el control del gasto de energía corporal es muy importante en el tratamiento de la obesidad.

\section{MATERIAL Y MÉTODOS}

Se realizó un estudio de intervención no randomizado y fueron seleccionadas $26 \mathrm{mu}$ jeres con exceso de peso corporal [índice de masa corporal (IMC)=peso $(\mathrm{kg}) /$ talla $\left(\mathrm{m}^{2}\right)$ $\geq 25 \mathrm{~kg} / \mathrm{m}^{2}$ ] (G1) y 14 eutróficas (IMC entre 18,5 y $24,9 \mathrm{~kg} / \mathrm{m}^{2}$ ) (G2), en el ambulatorio de dietoterapia de la División de Salud de la Universidad Federal de Viçosa (UFV) (MG)Brasil. Los criterios de inclusión consideraron la ausencia de pérdida de peso corporal de más de $3 \mathrm{~kg}$ en los últimos 3 meses, la ausencia de otras enfermedades crónicas, los no fumadores, sin el uso de medicamentos controlados y con la regla normal. Todas las mujeres fueron sometidas a análisis bioquímicos en sangre y orina para confirmar la adecuación de las mismas.

El estudio fue aprobado por el equipo de ética en investigaciones con seres humanos de la UFV (№ 059/2008).

Fue realizada la evaluación dietética ${ }^{21}$, la cual incluyó los datos de energía y macronutrientes. Para los análisis de estos parámetros se utilizó el programa de análisis de dietas DietPRO 5i, desarrollado por el Departamento de Nutrición y Salud de la UFV.

La evaluación del gasto energético en actividad física fue hecha por medio del acelerómetro tridimensional (Tritrac$\left.\mathrm{R}^{\circledR} \mathrm{D}^{\circledR}\right)^{22}$, utilizado por cada participante durante tres días. También se preguntó el tipo y la frecuencia del ejercicio físico y la regularidad del mismo ${ }^{23}$.

La evaluación antropométrica fue realizada en ayuno y incluyó las medidas de peso y talla corporales para calculo del $\operatorname{IMC}^{24}$ y circunferencia de la cintura (CC $)^{25}$. La composición corporal se determinó mediante el uso de la bioimpedancia eléctrica (BIA) (Biodinamic 310$)^{26}$, la cual se basa en el paso de la corriente eléctrica de baja frecuencia en el tejido hidratado, estimándose el agua corporal total (ACT), que refleja la MLG. Teniéndose en cuenta que el cuerpo está compuesto de dos compartimientos, la diferencia entre la masa corporal total y la MLG es la $\mathrm{MG}^{27}$.

El monitor metabólico de calorimetría indirecta (Deltatrac $\mathrm{II}^{\circledR}$ ) fue utilizado para la evaluación del metabolismo energético basal (durante 30 minutos, después de 12 horas en ayunas) y de reposo (durante 30 minutos, 3 horas y 30 minutos después de la comida). La calorimetría indirecta evalúa la tasa metabólica mediante la determinación del consumo de $\mathrm{O}_{2}$, con un espirómetro, y la producción estimada de $\mathrm{CO}_{2}$ procedentes del cuerpo, durante un período de tiempo determinado. Multiplicándose el peso corporal de los voluntarios por la TMB y TMR, se obtuvo, respectivamente, el GEB y el gasto energético en reposo (GER) ${ }^{12}$. La obtención del CR fue hecha considerando la tasa de oxidación de los macronutrientes, la cual se basó en la relación entre volúmenes de $\mathrm{CO}_{2} \mathrm{y}_{2}^{6,14}$.

Después de la evaluación basal, se ofreció una dieta líquida comercial normocalórica, normoproteica, normolipídica y normoglicídica ${ }^{28}(106 \mathrm{Kcal} / 100 \mathrm{~g}$, con un $61,13 \%, 24,20 \%$ y $15,09 \%$ de hidratos de carbono, lípidos y proteinas, respectivamente), con volumen equivalente a un $35 \%$ del gasto energético total (GET), para evaluación del metabolismo en reposo.

Entre las evaluaciones, las mujeres permanecieron en un ambiente tranquilo 
y cómodo, con actividad sedentaria. La temperatura y la humedad de ese ambiente fueron controlados ${ }^{12}$.

\section{Análisis estadísticos}

Los análisis estadísticos fueron desarrollados con la ayuda del software SAEG (sistema de análisis estadístico y genéticos), considerando el nivel de significancia de un $1 \%$ y $5 \%$ de probabilidad. Los datos se distribuyen normalmente. Para las comparaciones entre grupos se aplicó la prueba t no pareada. Para la evaluación de los parámetros metabólicos se consideró el diseño en bloques al azar con 26 y 14 bloques (G1 y G2, respectivamente) y 5 tratamientos (tiempo de evaluación en minutos), siendo realizado el análisis de varianza (ANOVA) y una regresión. La elección de los modelos fue basada en la importancia de los coeficientes de regresión, utilizando la prueba $t, y$ en los coeficientes de determinación. También se llevó a cabo el análisis de correlación de Pearson entre las variables antropométri- cas y metabólicas, considerándose $\mathrm{r}$ por debajo de $0,19,0,20$ a $0,39,0,40$ a $0,59,0,60$ a 0,79 y por encima de 0,80 como correlaciones no significativas, baja, moderada, fuerte y muy fuerte, respectivamente.

\section{RESULTADOS}

La edad de los grupos G1 y G2 fue de $36,62 \pm 7,76$ y $35,43 \pm 7,52$ años, respectivamente $(p>0,05)$. El IMC de G1 y G2 fue de $31,16 \pm 3,18$ y $21,77 \pm 1,86 \mathrm{~kg} / \mathrm{m}^{2}(\mathrm{p}<0,05)$, siendo clasificadas como eutróficas y con exceso de peso corporal (sobrepeso/obesidad), respectivamente, según la $\mathrm{OMS}^{24}$.

En la tabla 1 se representan los parámetros antropométricos y de composición corporal de G1 y G2. G1 presentó mayor CC $(\mathrm{p}<0,05)$, comparado con el G2. Además, la CC clasifica $\mathrm{G} 1$ en el riesgo muy alto de complicaciones metabólicas asociadas con la obesidad ${ }^{24}$. También la MG, MLG y GEB (evaluado por la BIA) fueron superiores en el G1, mientras que el G2 presentó un ACT mayor que el G1 $(\mathrm{p}<0,05)$.

Tabla 1. Comparación (media \pm DE) de los parámetros antropométricos y de la composición corporal entre G1 y G2.

\begin{tabular}{lcc}
\hline \multicolumn{1}{c}{ Parámetros } & G1 & G2 \\
\hline Circunferencia de la cintura (cm) & $96,78 \pm 8,78 \mathrm{a}$ & $73,81 \pm 5,29 \mathrm{~b}$ \\
Grasa corporal total (\%) & $38,32 \pm 4,00 \mathrm{a}$ & $27,88 \pm 4,38 \mathrm{~b}$ \\
Masa libre de grasa (kg) & $48,98 \pm 4,58 \mathrm{a}$ & $39,28 \pm 3,74 \mathrm{~b}$ \\
Agua corporal total (\%) & $43,17 \pm 2,80 \mathrm{a}$ & $51,54 \pm 3,61 \mathrm{~b}$ \\
Gasto energético basal (kcal) (Bioimpedancia eléctrica) & $1.488,58 \pm 139,08 \mathrm{a}$ & $1.194,71 \pm 113,47 \mathrm{~b}$ \\
\hline
\end{tabular}

Las medias seguidas por la misma letra en las filas no difieren a un $5 \%$ de probabilidad, por la prueba $t$. DE: desviación estándar.

Para la totalidad de la población estudiada, se observó una fuerte correlación positiva entre el IMC y la grasa corporal total $(\mathrm{GCT})(\mathrm{r}=0,79)$ y entre el GEB y la MLG $(\mathrm{r}=0,69)$, y una correlación baja entre el IMC y el GEB $(r=0,39)(p<0,05)$.

La ingesta habitual de energía no fue diferente $(\mathrm{p}>0,05)$ entre los grupos $\left(1.914,16_{ \pm}\right.$ 575,89 y $1.904,53 \pm 592,97 \mathrm{kcal}$ en el G1 y el $\mathrm{G} 2$, respectivamente). La ingesta de hidra- tos de carbono, lípidos y proteínas también fue semejante entre los grupos, siendo de $54,16 \pm 6,09 \%$ y $57,07 \pm 5,08 \%, 29,28 \pm 7,35 \%$ y $25,20 \pm 3,92 \%$ y, $16,68 \pm 3,63 \%$ y $17,72 \pm 3,47 \%$ en el G1 y el G2, respectivamente $(\mathrm{p}>0,05)$.

Se observó que el $38,46 \%$ y el $50 \%$ de las mujeres del G1 y del G2, respectivamente, eran sedentarias. La contribución del gasto energético en actividad en relación con el GET fue de un $33,04 \pm 7,76 \%$ y $30,43 \pm 6,65 \%$, 
y el factor de actividad física de un 1,51 \pm 0,16 y $1,44 \pm 0,13$ en G1 y G2, respectivamente, siendo mayor en el G1 $(p<0,05)$. Sin embargo, ambos grupos fueron clasificados como sedentarios ${ }^{29}$.
En la figura 1 (A y B) se presentan las estimaciones del $\mathrm{VCO}_{2}$ basal $\left(\mathrm{VCO}_{2} \mathrm{~B}\right)$ y en reposo $\left(\mathrm{VCO}_{2} \mathrm{R}\right)$ y $\mathrm{VO}_{2}$ basal $\left(\mathrm{VO}_{2} \mathrm{~B}\right)$ y en reposo $\left(\mathrm{VO}_{2} \mathrm{R}\right)$ del G1 y del G2, los cuales no fueron diferentes entre los grupos $(p>0,05)$.

Figura 1A. Valor estimado de los volúmenes de dióxido de carbono $\left(\mathrm{VCO}_{2}\right)$ y oxígeno $\left(\mathrm{VO}_{2}\right)$ en función del tiempo (minutos), en el período basal, en G1 y G2, respectivamente $\left(\mathrm{VCO}_{2} \mathrm{~B} 1\right.$ y VO $2 \mathrm{~B} 1, \mathrm{VCO}_{2} \mathrm{~B} 2$ y VO $\left.2 \mathrm{~B} 2\right)$.

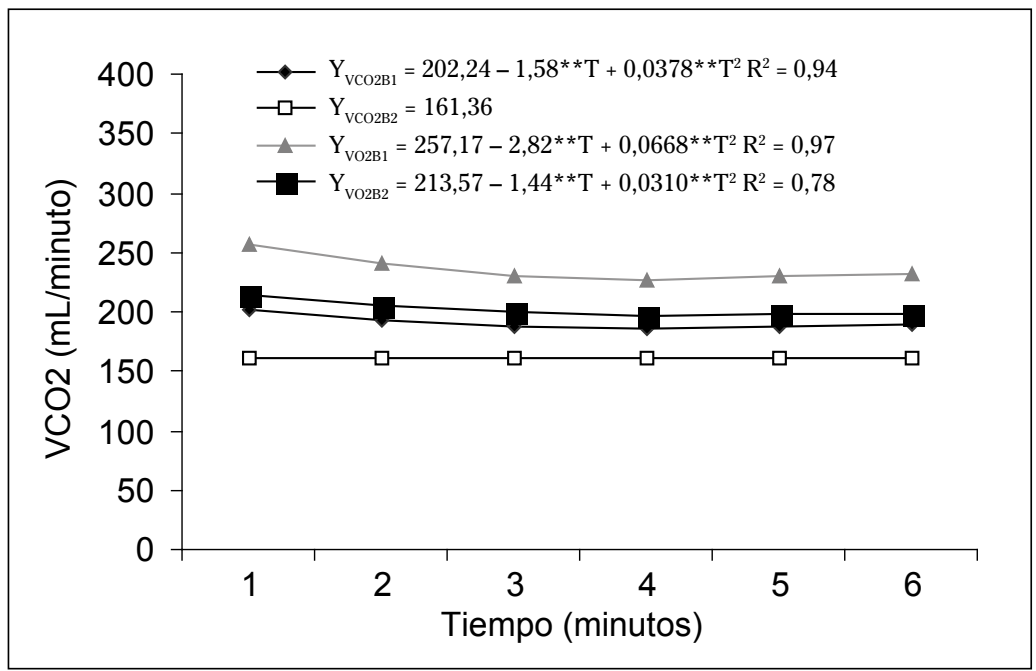

${ }^{*}{ }^{*}$ Significativo a un $1 \%$ de probabilidad, por la prueba $t$.

Figura 1B. Valor estimado de los volúmenes de dióxido de carbono $\left(\mathrm{VCO}_{2}\right)$ y oxígeno $\left(\mathrm{VO}_{2}\right)$ en función del tiempo (minutos), en el período de reposo, en G1 y G2, respectivamente $\left(\mathrm{VCO}_{2} \mathrm{R} 1\right.$ y VO $2 \mathrm{R} 1, \mathrm{VCO}_{2} \mathrm{R} 2$ y VO $\left.2 \mathrm{R} 2\right)$.

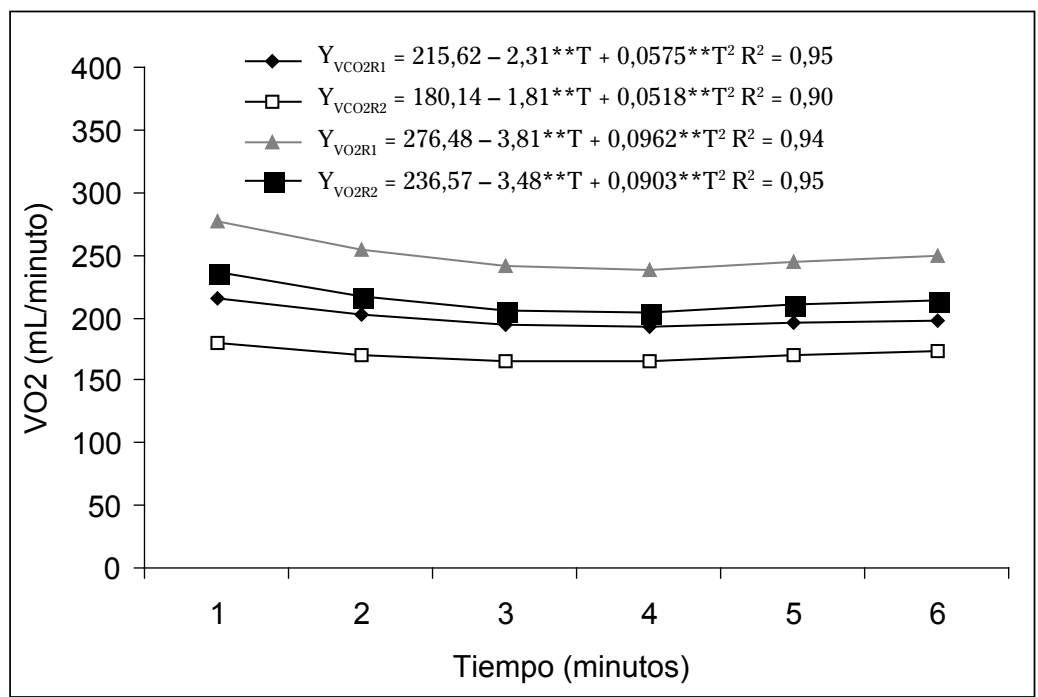

* Significativo a un $1 \%$ de probabilidad, por la prueba $t$. 
El $\mathrm{VCO}_{2} \mathrm{~B}$ presentó un valor cuantitativo superior en el G1 $(189,07 \pm 22,50$ y $159,53 \pm$ $17,82 \mathrm{ml} / \mathrm{min}$ en el G1 y G2, respectivamente) $(\mathrm{p}>0,05)$. En el G2, el $\mathrm{VCO}_{2} \mathrm{~B}$ fue constante y en el G1 el mismo disminuyó y, posteriormente, aumentó. También para el $\mathrm{VO}_{2} \mathrm{~B}$, el G1 mostró una tendencia de valores más elevados $(233,57 \pm 20,48$ y 198,31 $\pm 21,68$ $\mathrm{ml} / \mathrm{min}$ en el G1 y el G2, respectivamente), pero sin significancia estadística $(p>0,05)$, existiendo disminución de ambos, los cuales alcanzaron valores mínimos a los 21,14 $(227,31 \mathrm{ml} / \mathrm{min})$ y 23,26 minutos $(196,81 \mathrm{ml} /$ min), en el G1 y el G2, respectivamente (Fig. 1A).

La tendencia hacia valores superiores en el G1 también fue observada en las variables $\mathrm{VCO}_{2} \mathrm{R}$ y $\mathrm{VO}_{2} \mathrm{R}$. Para $\mathrm{VCO}_{2} \mathrm{R}$ se obtuvieron valores mínimos de 196,76 $\pm 19,49$ y $165,15 \pm 18,36 \mathrm{ml} / \mathrm{min}$ en el G1 y el G2, respectivamente $(\mathrm{p}>0,05)$. En ambos grupos hubo reducción del último parámetro, el cual alcanzó valores mínimos a los 20,04 $(192,52 \mathrm{ml} / \mathrm{min})$ y 17,40 minutos $(164,45$ $\mathrm{ml} / \mathrm{min}$ ), en el G1 y el G2, respectivamente. Para $\mathrm{VO}_{2} \mathrm{R}$, los valores fueron de 247,38 $\pm 20,26$ y $207,23 \pm 18,84 \mathrm{ml} / \mathrm{min}$ en el G1 y el G2, respectivamente ( $p>0,05)$, y ambos muestran un descenso hasta alcanzar el valor mínimo a los $19,81(238,73 \mathrm{ml} / \mathrm{min})$ y 19,26 minutos (203,07 ml/minuto) (Fig. 1B).

En la figura 2 se presentan las estimaciones de los valores de CR basal (CRB) y en reposo (CRR), en el G1 y el G2. El G1 presentó tendencia a CRB superior $(0,81 \pm 0,04$ y $0,80 \pm 0,03 \mathrm{ml} / \mathrm{min}$ en el G1 y G2, respectivamente) $(p>0,05)$. Al final de la evaluación, los grupos tuvieron valores similares, y el G1 y el G2 alcanzaron el máximo CRB a los 23,23 $\left(0,81\right.$ moles de $\left.\mathrm{CO}_{2} / \mathrm{mol} \mathrm{O}_{2}\right)$ y 22,12 minutos $\left(0,82\right.$ moles de $\left.\mathrm{CO}_{2} / \mathrm{mol} \mathrm{O}_{2}\right)$, respectivamente. El CRR fue similar entre los grupos $(0,80 \pm 0,05$ y $0,80 \pm 0,03 \mathrm{ml} / \mathrm{min}$ en el G1 y el G2, respectivamente) $(\mathrm{p}>0,05)$. El máximo de CRR se alcanzó a los 19,58 $\left(0,81\right.$ moles de $\left.\mathrm{CO}_{2} / \mathrm{mol} \mathrm{O}_{2}\right)$ y 23,80 minutos $\left(0,81\right.$ moles de $\left.\mathrm{CO}_{2} / \mathrm{mol} \mathrm{O}_{2}\right)$ en el G1 y el G2, respectivamente.

Figura 2. Valor estimado del cociente respiratorio basal (CRB) y reposo (CRR), en función del tiempo (minutos), en G1 (CRB1 y CRR1) y G2 (CRB2 y CRR2), respectivamente.

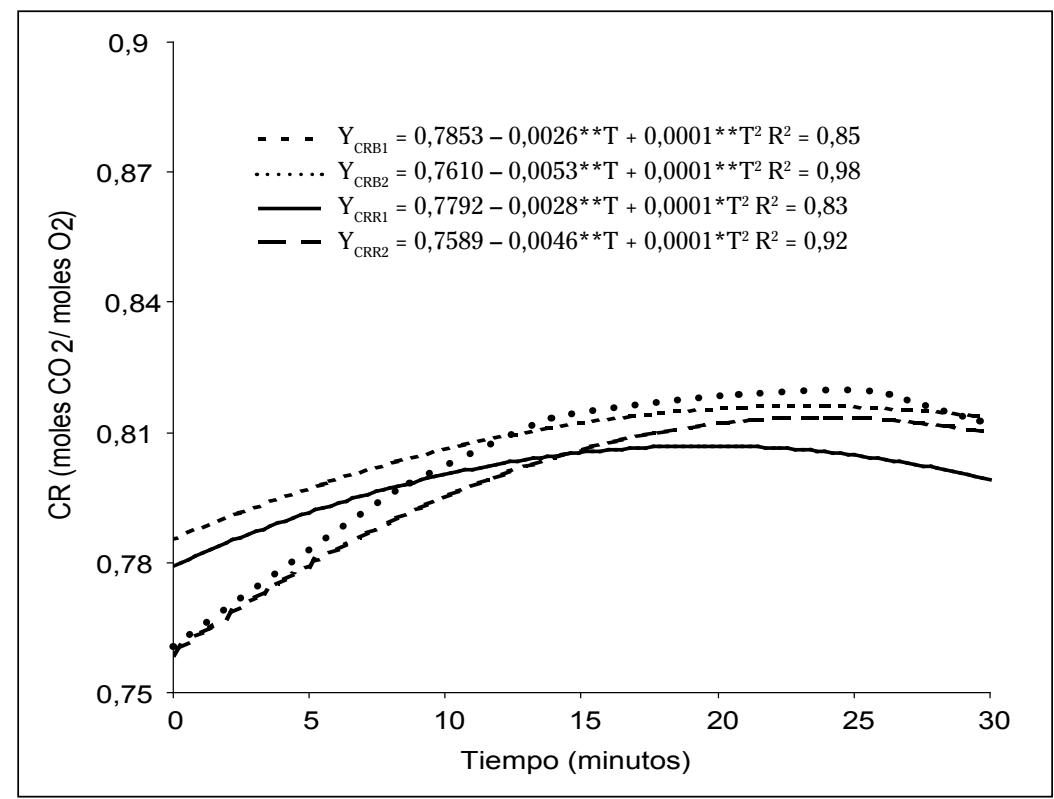

* Significativo a un 5\% de probabilidad, por la prueba t.

* * Significativo a un $1 \%$ de probabilidad. 
No se encontraron correlaciones significativas entre el CR y el peso corporal $(p>0,05)$.

El GEB y GER del G1 y del G2 son presentados en la figura 3. El G1 mostró valores absolutos más altos, tanto en el momento basal cuanto en el reposo ( $>0,05)$ (GEB: $1591,54 \pm 148,96$ y $1345,38 \pm 148,47 \mathrm{ml} / \mathrm{mi}-$ nuto, y GER: $1.680,38 \pm 139,01$ y $1405,85 \pm$ $155,26 \mathrm{ml} / \mathrm{min}$ en $\mathrm{G} 1$ y G2, respectivamen- te). El GEB mínimo fue logrado a los 21,10 minutos $(1.550,13 \mathrm{kcal})$ en G1 y $11,10 \mathrm{minu}-$ tos $(1.347,47 \mathrm{kcal})$ en el G2, en el cual se observó el cambio en la tendencia de la curva, con un aumento a los 16,82 minutos y un valor máximo a los 22,54 minutos $(1.369,26 \mathrm{kcal})$. Para el GER, el valor mínimo fue verificado a los 19,88 (1.625,60 kcal) y 18,96 minutos $(1.380,50 \mathrm{kcal})$ en $\mathrm{G} 1$ y G2, respectivamente $(\mathrm{p}>0,05)$.

Figura 3. Valor estimado del gasto energético basal (GEB) y en reposo (GER), en función del tiempo (minutos), en G1 (GEB1 y GER1) y G2 (GEB2 y GER2).

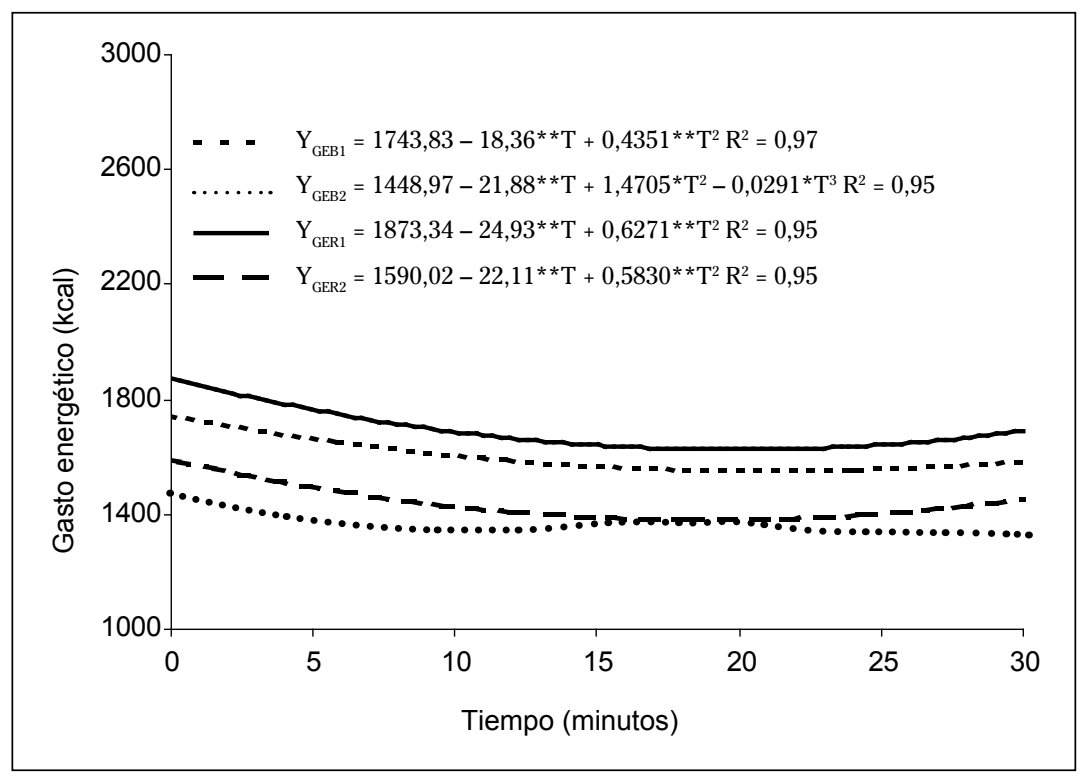

* Significativo a un $5 \%$ de probabilidad, por la prueba t.

* * Significativo a un $1 \%$ de probabilidad.

\section{DISCUSIÓN}

La CC refleja la cantidad de grasa visceral, la cual se relaciona con el desarrollo de enfermedades crónicas no transmisibles, particularmente la cardiovasculares ${ }^{30}$. En este estudio, el G1 tuvo mayor riesgo de complicaciones metabólicas asociadas a la obesidad $^{24}$.

El G1 presentó mayor masa corporal total, GCT, MLG y GEB (evaluado por la BIA) $(\mathrm{p}<0,05)$. Hallazgos anteriores han demostrado que la obesidad se caracteriza por el aumento de MG y MLG ${ }^{31}$. El mayor GEB en el G1 se debe al hecho de que el aparato BIA no considera las diferencias en la oxidación de sustratos entre los grupos, como se evidencia en la calorimetría indirecta. Por lo tanto, las fórmulas teóricas que evalúan el GEB pueden sobrestimar el parámetro en obesos.

Marks y Rippe ${ }^{7}$ indicaron que las personas obesas presentan el músculo esque- 
lético aumentado, como se verificó en el presente estudio pero con menor densidad y disminución de la eficiencia, debido a la menor capilarización y densidad mitocondrial, lo que resulta en la pérdida de la capacidad de trabajo y variación en el contenido de agua de la MLG.

En obesos, la TMB puede no ser directamente proporcional a la MLG. Una explicación para este hecho es que en la obesidad los órganos metabólicamente activos no se hipertrofian, o sea, el aumento ocurre en escala más baja, comparada con la masa muscular, lo que cambia la composición de la MLG. Por lo tanto, la TMB depende de la cantidad y de la actividad metabólica de la masa celular corporal, así como de su proporción en relación a la $\mathrm{MLG}^{15}$. A pesar de la sugerencia de que la TMB sea expresada en función de la masa magra y no de la masa corporal total ${ }^{32}$ siguen siendo pocos los datos disponibles de TMB y de la composición corporal en obesos, debido, principalmente, a la limitación metodológica para medir la composición corporal ${ }^{33}$. Por otro parte, hay estudios que confirman que el GEB superior en obesos puede ser explicado por la mayor cantidad de MLG $^{31-32}$.

En el presente estudio, la MLG se asoció con el GEB. Además, la MLG fue el principal determinante del GEB, en comparación con el IMC, en las mujeres con exceso de peso corporal.

El ACT es el componente cuantitativamente más importante de la MLG. Sartorius y col $^{34}$ observaron que las mujeres obesas tenían más ACT, en comparación con las no obesas (con peso normal y sobrepeso), sin diferencia entre los grados de obesidad. En el presente estudio, la ACT fue mayor en el G2; sin embargo, nuestro grupo sólo incluyó a las mujeres eutróficas.

Los parámetros dietéticos sugieren la subestimación de la ingesta en el G1 pues, a pesar de la ausencia de pérdida de peso, la ingesta de energía estuvo por debajo de los requerimientos de energía para el mantenimiento del peso corporal. Este hecho es muy común en la evaluación de la dieta de individuos con exceso de peso corporal, cuando a menudo lo que se calcula en rela- ción a la ingesta se encuentra por debajo de los niveles recomendados, una condición conocida como "flat-slope syndrome» ${ }^{35}$.

Los componentes del G1 y del G2 eran sedentarios. Así, el gasto de energía en la actividad física no tuvo efecto sobre la composición corporal y el metabolismo energético de las mujeres estudiadas. Cabe señalar que el trabajo muscular de baja intensidad conduce principalmente a la oxidación de hidratos de carbono ${ }^{36}$, la cual tiende a ser mayor en sujetos con exceso de peso ya que muestran tendencia a ahorrar energía, además de su mayor eficiencia metabólica ${ }^{37}$.

Existen hipótesis sobre los posibles cambios en la utilización de los macronutrientes en personas obesas ${ }^{14}$, en los cuales se sugiere la menor oxidación de los lípidos y el aumento en la oxidación de hidratos de carbono. Sin embargo, Mourão y col $^{38}$ no observaron diferencia en la evaluación del CR en hombres eutróficos (IMC $=23,04$ $\pm 0,50 \mathrm{~kg} / \mathrm{m}^{2}$ ) y con sobrepeso (IMC $=29,05$ $\left.\pm 0,60 \mathrm{~kg} / \mathrm{m}^{2}\right)$, después de la ingesta de una dieta isoenergética estándar y modificada (diet). Los autores atribuyeron el resultado a la reducida GCT en el grupo con sobrepeso. En nuestro estudio, es posible sugerir que las mujeres con exceso de peso presentaron tendencia a una mayor eficiencia metabólica, en comparación con las mujeres eutróficas, basado en el comportamiento de las curvas del CR.

Prentice y $\mathrm{col}^{37}$ observaron que la predisposición a la obesidad puede ser producto del metabolismo más económico que busca mantener la masa corporal estable. Ravussin y col $^{28}$ encontraron que los individuos con características físicas similares pueden requerir más o menos energía para mantener su peso corporal y pueden presentar diferencias en la eficiencia metabólica. En este sentido, a pesar de verificar en nuestro estudio que el G1 alcanzó el $\mathrm{VO}_{2} \mathrm{~B}$ mínimo más rápido, no hubo diferencia estadística entre los grupos. Esto sugiere que el G1 presente mayor reducción en la oxidación de los nutrientes, probablemente dirigida al mantenimiento de las reservas de grasa, pero el $\mathrm{VO}_{2}$ se relaciona 
con mayor gasto de energía, que, a su vez, se correlaciona con el IMC ${ }^{39}$. Por lo tanto, el presente resultado sugiere que en el G1 hubo una compensación de la reducción en la oxidación de los nutrientes por la mayor masa corporal, lo que resultó en $\mathrm{VO}_{2} \mathrm{~B}$ semejante entre el G1 y el G2.

El $\mathrm{VCO}_{2}$ se relaciona con el tipo de nutriente oxidado y la eficiencia metabólica ${ }^{13}$. El $\mathrm{VCO}_{2} \mathrm{R}$ refleja el metabolismo postabsortivo. El presente estudio no verificó diferencia en el metabolismo postabsortivo entre los grupos después de una comida con cantidades de energía y lípidos normales.

El CRR máximo, en valores absolutos, sugieren que G1 presentó mayor oxidación de hidratos de carbono, en comparación con el G2. Sin embargo, ambos mostraron una mayor oxidación de hidratos de carbono en ayuno y en reposo ya que el CR se mantuvo por encima de $0,75^{40}$.

Marra y $\mathrm{col}^{41}$ observaron una fuerte correlación entre el CR y el peso corporal. También Martínez y col ${ }^{42}$ afirmaron que la obesidad puede asociarse al cambio en la utilización de los nutrientes, con baja prioridad en la oxidación de lípidos. Ya Ravussin y col $^{43}$ observaron que el CR de 24 horas fue de 0,82 en individuos eutróficos, 0,84 en aquellos moderadamente obesos y $0,81$ en obesos ( $p>0,05)$, lo que confirma las conclusiones del presente estudio, ya que no se observaron correlaciones entre estas variables.

Valtueña y col $^{14}$ evaluaron el metabolismo energético en sujetos obesos de grado 3 y observaron que aquellos que presentaban bajo CR, como resultado del aumento de la oxidación de los lípidos, eran más capaces de mantener el peso corporal después de una dieta hipocalórica. Buscemi y col$^{27}$ observaron un CR más bajo en sujetos obesos de grado 3, en comparación con los eutróficos. Las mujeres evaluadas en el presente estudio eran en su mayoría obesas de grado 1, con menor cantidad de grasa, cuando comparadas con el estudio citado.

Cabe señalar que los estudios son aún contradictorios con respecto a la relación del CR con la masa corporal. Walewski y col (2010) ${ }^{44}$ afirmaron que los obesos presentan mayor cantidad de tejido adiposo omental, comparado con los controles, y la expresión de genes claves involucrados en el lipólisis, beta-oxidación y metabolismo de los ácidos grasos está reducida.

El GEB y el GER no presentaron diferencia entre los grupos y, considerándose que los mismos fueron calculados utilizándose la TMB y la TMR, respectivamente, multiplicado por el peso corporal, se sugiere que el gasto de energía podría ser superior en G1. Sin embargo, debido al exceso de MG, metabólicamente menos activa, el GEB y el GER son inferiores a los valores previstos para la masa corporal total. La reducción de la TMB y de la TMR en individuos con exceso de peso corporal puede conducir a una sobreestimación del GEB y del GER obtenidos por medio de fórmulas teóricas.

En el presente estudio, las mujeres con exceso de peso corporal presentaron mayor GCT, MLG y CC, con riesgo muy elevado para la ocurrencia de complicaciones metabólicas asociadas con la obesidad. Además, la ingesta dietética habitual y la actividad física fueron semejantes entre las mujeres con exceso de peso corporal y las eutróficas. Los parámetros metabólicos sugieren una mayor eficiencia metabólica y menor oxidación de lípidos en las mujeres con exceso de peso corporal, pero estos resultados no son concluyentes. La composición corporal se relacionó con el gasto de energía, ya que la MLG fue el principal determinante del GEB.

Se concluye que la obesidad puede estar relacionada con alteraciones del gasto energético corporal, lo cual es influenciado por la composición corporal. El aumento de la grasa corporal resulta en reducción del gasto energético, ya que ocurre una reducción proporcional del tejido metabólicamente activo, lo que puede llevar al desequilibrio energético y, por lo tanto, al aumento del peso corporal.

Son necesarios nuevas investigaciones para evaluar los factores que intervienen en la reducción del gasto energético y en la oxidación de los macronutrientes, los cua- 
les darán soporte para las intervenciones dietéticas personalizadas, teniéndose en cuenta los cambios en la eficiencia metabólica en individuos con obesidad.

\section{Agradecimientos \\ FAPEMIG (Fundação de Amparo à Pes- quisa do Estado de Minas Gerais) por la ayuda financiera. Universidade Federal de Viçosa, por la oportunidad de realización de la investigación. Al laboratorio Santa Rita (Viçosa-MG) por los análisis de labo- ratorio. Abbott por la dietas comerciales.}

\section{BIBLIOGRAFÍA}

1. Rosado El, Bressan J, Martins MF, Cecon PR, Martínez JA. Polymorphism in the PPARgamma2 and beta2-adrenergic genes and diet lipid effects on body composition, energy expenditure and eating behavior of obese women. Appetite 2007; 49: 635-643.

2. FLATT JP. Glycogen levels and obesity. Int J Obes Relat Metab Disord 1996; 20 (Suppl. 2): S1-11.

3. Kim JY, Hickner RC, Cortright RL, Dohm GL, HoumARD JA. Lipid oxidation is reduced in obese human skeletal muscle. Am J Physiol Endocrinol Metab 2000; 279: E1039-1044.

4. HA UFE S, EN GELI S, BüDZIAREK P, UT Z W, S HULZ-MENGER J, HermsDORF M ett al. Determinants of exercise-induced fat oxidation in obese women and men. Horm Metab Res 2010; 42: 215-221.

5. Horowitz JF, KLEIN S. Whole body and abdominal lipolytic sensitivity to epinephrine is suppressed in upper body obese women. Am J Physiol Endocrinol Metab 2000; 278: E11441152.

6. NAGy TR, Goran MI, Weinsier RL, Toth MJ, Schutz Y, Poehlman ET. Determinants of basal fat oxidation in healthy caucasians. J Appl Physiol 1996; 80: 1743-1748.

7. MARKs BL, RiPPE JM. The importance of fat free mass maintenance in weight loss programmes. Sports Med 1996; 22: 273-281.

8. Stiegler P, Cunliffe A. The role of diet and exercise for the maintenance of fat-free mass and resting metabolic rate during weight loss. Sports Med 2006; 36: 239-262.

9. CEDDIA RB. Gordura corporal, exercício e emagrecimento. Reviews Sprint Magazine 1998; 1: 10-20.
10. Astrup A, Buemann B, Western P, Toubro S, RABen A, Christensen NJ. Obesity as an adaptation to a high-fat diet: evidence from a cross-sectional study. Am J Clin Nutr 1994; 59: 350-355.

11. Hermana HMH, Volp ACP, Bressan J. O perfil de macronutrientes influencia a termogênese induzida pela dieta e a ingestão calórica. ALAN 2007; 57: 33-42.

12. FERRANNINI E. The theoretical bases of indirect calorimetry: a review. Metabolism 1988; 37: 287-301.

13. Westerterp-Plantega MS, WiJCKMANS-DuIJSENS NEG, Verboeket-Van de Venne WPG, GraAF KH, WESTSTRATE JA. Energy intake and body weight effects of six months reduced or full fat diets, as a function of dietary restraint. Int $\mathrm{J}$ Obes 1998; 22: 14-22.

14. Valtueña S, Salas-Salvadó J, Lorda PG. The respiratory quotient as a prognostic factor in weight-loss rebound. Int J Obes 1997; 21: 811-817.

15. Censi L, Toti E, Pastore G, Ferro-Luzzi A. The basal metabolic rate and energy cost of standardized walking of short and tall men. Eur J Clin Nutr 1998; 52: 441-446

16. GORAN MI. Energy metabolism and obesity. Med Clin North Am 2000; 84: 347-362.

17. DeJong AT, Gallagher MJ, Sandberg KR, LillystoNE MA, Spring T, Franklin BA et al. Peak oxygen consumption and the minute ventilation/ carbon dioxide production relation slope in morbidly obese men and women: influence of subject effort and body mass index. Prev Cardiol 2008; 11: 100-105.

18. Jacobson P, Rankinen T, Tremblay A, Pérusse L, Chagnon YC, Bouchard C. Resting metabolic rate and respiratory quotient: results from a genome-wide scan in the Quebec family study. Am J Clin Nutr 2006; 84: 1527-1533.

19. Marra M, Pastisi F, Montagnese C, DE FilipPo E, DE CAPRIO C, DE MAGISTRIS L eldal. BMR variability in women of different weight. Clin Nutr 2007; 26: 567-572.

20. Katzmarzyk PT, Pérusse L, Tremblay A, Bouchard C. No association between resting metabolic rate or respiratory exchange ratio and subsequent changes in body mass and fatness: 5-1/2 year follow-up of the Québec family study. Eur J Clin Nutr 2000; 54: 610-614.

21. Fontanive RS, De Paula TP, Peres WAF. Inquéritos dietéticos. En: Duarte AC, Castellani FR. Semiologia nutricional. Rio de Janeiro: Axcel Books 2002, 4; 59-78. 
22. Mathews CE, FREedson PS. Field trial of a threedimensional activity monitor: comparison with self report. Med Sci Sports Exerc 1995; 27: 1071-1078.

23. World Health Organization. Physical status: the use and interpretation of anthropometry. Report of a WHO Expert Committee. Technical Report Series 854. Geneva: WHO, 1995.

24. World Health Organization. Obesity: preventing and managing the global epidemic. Report of a WHO Consultation on Obesity. WHO Technical Report Series 894. Geneva: WHO, 1998.

25. Consenso Latino Americano de Obesidade, 1999, 117 p. Disponível no endereço eletrônico da Associação Brasileira de Estudos Sobre Obesidade, URL http://www.abeso.org. br (Acesso el 03/10/2007).

26. LUKASKI HC, JOHNSON PE, BolONCHUK WW, LYKKEN GI. Assessment of fat-free mass using bioelectrical impedance measurements of the human body. Am J Clin Nutr 1985; 41: 810-817.

27. Buscemi S, Caimi G, Verga S. Resting metabolic rate and postabsorptive substrate oxidation in morbidly obese subjects before and after massive weight loss. Int J Obes 1996; 20: 4146.

28. Ravussin E, Lillioja S, Knowler WC, Christin L, FREYMOND D, AввоTt WG et al. Reduced rate of energy expenditure as a risk factor for bodyweight gain. N Engl J Med 1988; 318: 467-472. World Health Organization. Energy and protein requirements. Report of a Joint FAO/ WHO/ONU Expert Consultation. Technical Report Series 724. Geneva: WHO, 1985.

29. Després JP, Lemieux I, Prud`homme D. Treatment of obesity: need to focus on high risk abdominally obese patients. BMJ 2001; 322: 716720 .

30. Lichtenbelt WD, Fogelholm M. Increased extracellular water compartment, relative to the intracellular water compartment, after weight reduction. J Appl Physiol 1999; 87: 294-298.

31. Piers LS, Soares MJ, McCormack LM, O’Dea K. Is there evidence for an age-related reduction in metabolic rate? J Appl Physiol 1998; 85: 2196-2204.

32. Warlich V, Anjos LA. Aspectos históricos e metodológicos da medição e estimativa da taxa metabólica basal: uma revisão da literatura. Cadernos de Saúde Pública 2001; 17 : 801-817.
33. Sartorio A, Malavolti M, Agosti F, Marinone PG, Caiti O, Battistini N et al. Body water distribution in severe obesity and its assessment from eight-polar bioelectrical impedance analysis. Eur J Clin Nutr 2005; 59: 155-160.

34. Roth J, Qiang X, Marbán SL, Redelt H, Lowell BC. The obesity pandemic: Where have we been and are we going? Obes Res 2004; 12 (Supl.): 88-101.

35. Curi R, Lagranha CJ, G Jr JR, Pithon-Curi TC, Lancha JR AH, Pellegrinotti IL et al. Ciclo de krebs como fator limitante na utilização de ácidos graxos durante o exercício aeróbico. Arq Bras Endocrinol Metab 2003; 47: 135-143.

36. Prentice AM, Rayco-Solon P, Moore SE. Insights from the developing world: thrifty genotypes and thrifty phenotypes. Proc Nutr Soc 2005; 64: 153-161.

37. Mourão DM, MonteIro JBR, HermsdorfF HHM, TEIXEIRA MCL Alimentos modificados e suas implicações no metabolismo energético. Rev Nutr 2005; 18: 19-28.

38. De Jong AT, Gallagher MJ, Sandberg KR, LillystoNE MA, Spring T, Franklin BA et al. Peak oygen consumption and the minute ventilation/ carbon dioxide production relation slope in morbidly obese men and women: Influence of subject effort and body mass index. Prev Cardiol 2008; 11: 100-105.

39. Flatt JP. Carbohydrate-fat interactions and obesity examined by a two-compartment computer model. Obes Res 2004; 12: 20132022.

40. Marra M, Scalfi L, Covino A, Esposito-Del Puente A, ConTALDo F. Fasting respiratory quotient as a predictor of weight changes in non obese women. Int J Obes 1998; 22: 601-603.

41. Martínez JA, Moreno MJ, Marqués-Lopes I, Martí A. Causas de obesidad. An Sist Sanit Navar 2002; 25 (Supl.1): 17-27.

42. Ravussin E, Burnand B, Schutz Y, JÉquier E. Twenty-four-hour energy expenditure and resting metabolic rate in obese, moderately obese, and control subjects. Am J Clin Nutr 1982; 35: 566-573.

43. WAL EWSKI JL, GE F, GAd NER M, INA BNET WB, Pom A, BRANCH AD et al. Adipocyte accumulation of long-chain fatty acids in obesity is multifactorial, resulting from increased fatty acid uptake and decreased activity of genes involved in fat utilization. Obes Surg 2010; 20: 93107 . 
